\title{
Interior Design in Augmented Reality Environment
}

\author{
Viet Toan Phan \\ Ph. D Candidate \\ ${ }^{1}$ School of Architecture \& Civil engineering \\ Kyungpook National University, Republic of Korea \\ ${ }^{2}$ Department of Industrial Construction \\ HaNoi University of Science Technology, VietNam
}

\author{
Seung Yeon Choo \\ Assistant Professor \\ School of Architecture \& Civil Engineering \\ Kyungpook National University, \\ Republic of Korea
}

\begin{abstract}
This article presents an application of Augmented Reality technology for interior design. Plus, an Educational Interior Design Project is reviewed. Along with the dramatic progress of digital technology, virtual information techniques are also required for architectural projects. Thus, the new technology of Augmented Reality offers many advantages for digital architectural design and construction fields. AR is also being considered as a new design approach for interior design. In an AR environment, the virtual furniture can be displayed and modified in real-time on the screen, allowing the user to have an interactive experience with the virtual furniture in a real-world environment. Here, AR environment is exploited as the new working environment for architects in architectural design works, and then they can do their work conveniently as such collaborative discussion through AR environment. Finally, this study proposes a new method for applying AR technology to interior design work, where a user can view virtual furniture and communicate with 3D virtual furniture data using a dynamic and flexible user interface. Plus, all the properties of the virtual furniture can be adjusted using occlusion- based interaction method for a Tangible Augmented Reality.
\end{abstract}

\section{General Terms}

Applications of computer science in modeling, visualization and multimedia, graphics and imaging, computer vision, humancomputer interaction, et al.

\section{Keywords}

Augmented Reality, Tangible AR, CAAD, ARToolKit, Interior design.

\section{INTRODUCTION}

Visualizing how a particular table or chair will look in a room before it is decorated is a difficult challenge for anyone. Hence, Augmented Reality (AR) technology has been proposed for interior design applications by few previous authors, for example, Koller, C. Wooward, A. Petrovski; K. Hirokazu, et al. The related devices typically include data glasses connected to a portable PC (Head-mounted display- HMD). Plus, various lightweight solutions using a PDA device has been proposed by the Augmented Reality Team in Findland (S. Sitanen and C. Woodward, 2003). However, these devices are right not commonly available for non-professional users.

Accordingly, this paper presents an augmented reality system for designing/educating/presenting interior design projects using overlaid virtual furniture in a physical environment based on a regular PC home system. Tracking markers are placed on the floors or walls to define the scale and coordinate system of the room. Next, the user selects virtual furniture on the screen and places it in the design space. In the AR scene, the $3 \mathrm{D}$ virtual furniture is integrated into a real environment and can be arranged along side real furniture. Experiments are implemented using basic home computer equipment, including a PC, HMD (or web camera), and printer. As a result, it is hoped that the proposed system will allow a broad range of users.

While some similar systems have already been presented by another research group, the system proposed in this paper includes additional functions for the user interface and an improved implementation. For example, the user can interact with virtual furniture using a Tangible Augmented Reality in real time, and change the color, style, or covering of furniture in a real environment. Therefore, this allows complex and varied designs to be explored and visualized, making AR technology for interior design accessible to both professionals and amateurs.

\section{AUGMENTED REALITY- NEW RESEARCH APPROACH FOR ARCHITECTURE}

\subsection{Augmented Reality technology}

Augmented Reality (AR) is a new technology that involves the overlay of computer graphics on the real world. As a result, the user can see the real world augmented with virtual objects and can interact with them. Within a more general context, AR is also termed Mixed Reality (MR), referring to a multi-axis spectrum of areas that cover Virtual Reality (VR), Augmented Reality (AR), telepresence, and other related technology [1] (Figure 1).

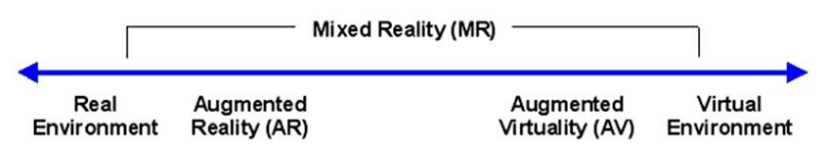

Figure 1. Paul Milgram's Reality- Virtual continuum.

Augmented Reality systems combine digital information and the real world in a way that the user experiences them as one. A particularly important property of AR is locating virtual objects in the right place and position, which makes the Tracking System one of the most important components of an AR system. Essentially, an AR system must be able to follow the user's point of view dynamically and keep virtual objects aligned with real world objects. The basic components of an AR system are a display, camera for graphic captures, and computer installed application software, plus various different kinds of hardware can 
be used, for example, camera phones, PDAs, lap-tops, HMDs, and wearable computer systems.

Typically, an ARToolKit library is used to determine the relation between the real and virtual world. The ARToolKit uses a computer vision technique to define the position and orientation of the real camera viewpoint relative to a real world marker. Next, the ARToolKit defines and calculates the position of the virtual coordinates. Based on a concurrence of virtual and real camera coordinates, the computer graphics are then drawn as an overlay on a fiducial markers card. As a result, the user experiences a video see-through augmented reality on the PC screen or more lively impression by HMDs (Kato, H.2001 and HIT Lab Washington University).

Although Augmented Reality has only been studied for one decade, the growth and progress in the past few years have been remarkable. As such, AR technology has many possible applications across a wide range of fields, including entertainment, education, medicine, military training, engineering, and manufacturing [2](Figure 2; 3).
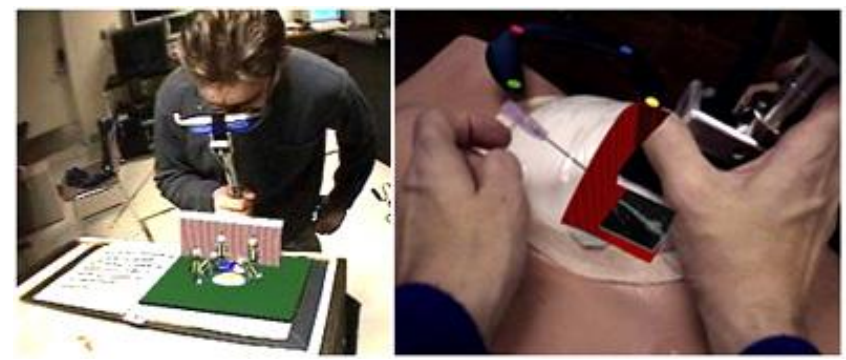

Figure 2. AR application in entertainment and medical fields.

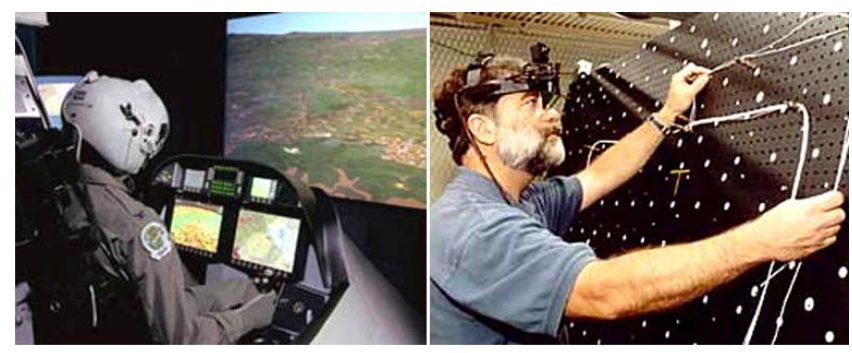

Figure 3. AR application in military and manufacture engineering fields.

It is also expected that other potential areas for application will still appear with the dissemination of this technology. During the early stages, the main focus of AR development was related to hardware technology rather than usability. However, the rapid development of mobile (handheld) with better processing capacities and long-lasting batteries has raised the issue of lightweight mobile AR systems. Thus, mobile AR devices are now one of the most promising emerging technologies. Similarly, the proposed system was also designed to appeal to a broader range of users based on the use of a regular PC and HMD.

\subsection{Augmented Reality in architecture field}

Recently, AR technology is also being considered as a new design approach for architecture. As a result, a lot of AR experiments and research have been directed toward the architectural design process. For example, Figure 4 Left shows a full-size 3D virtual house in a real life environment, where the handheld AR device allows the user to walk around and through it [3](Augmented Team- Finland 2003). Meanwhile, Figure 4- Right shows another implementation of AR in archaeology and touring guide, where the user is shown the virtual heritage buildings raised up from ruins on historical site.

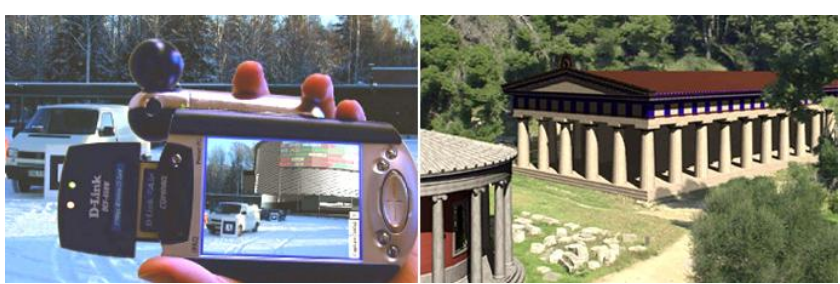

Figure 4. Left: Virtual building in PDA. Right: Virtual Hera temple in historical.

In the case of architecture, the above applications can be effective for both designing and teaching. However, a growing number of new applications of AR technology are expected in the field of architecture.

\section{INTERIOR DESIGN IN DIGITAL ENVIRONMENT \\ 3.1 Properties of interior design}

In the case of interior design, the designer essentially applies the three basic principles of interior design: color, scale, and proportion within a predetermined space. Thus, the proposed AR system is focused on giving the user the flexibility to design using these three basic principles. Therefore, in the proposed AR environment, the user is able to adjust the properties of virtual furniture and create different arrangements in a real environment.

\subsection{System design}

For implementation, two separate modules were developed: one for creating and managing the $3 \mathrm{D}$ database, and the other for displaying, as show in below figure (Figure 5).

First, CAD applications extract information from a drawing and link it to a database. For the given space, geometrical information is then extracted from a three-dimensional database of furniture. After loading the geometries, the position and direction of the views for the user are calculated based on data marker tracking. Simultaneously, the location- and direction- based geometry data are transformed using transformation matrices to produce images that align beside other objects in the real view. As such, the position tracker and orientation tracker are important elements of AR systems and the development AR technology. Figure 6 summaries the tracking and display process. 


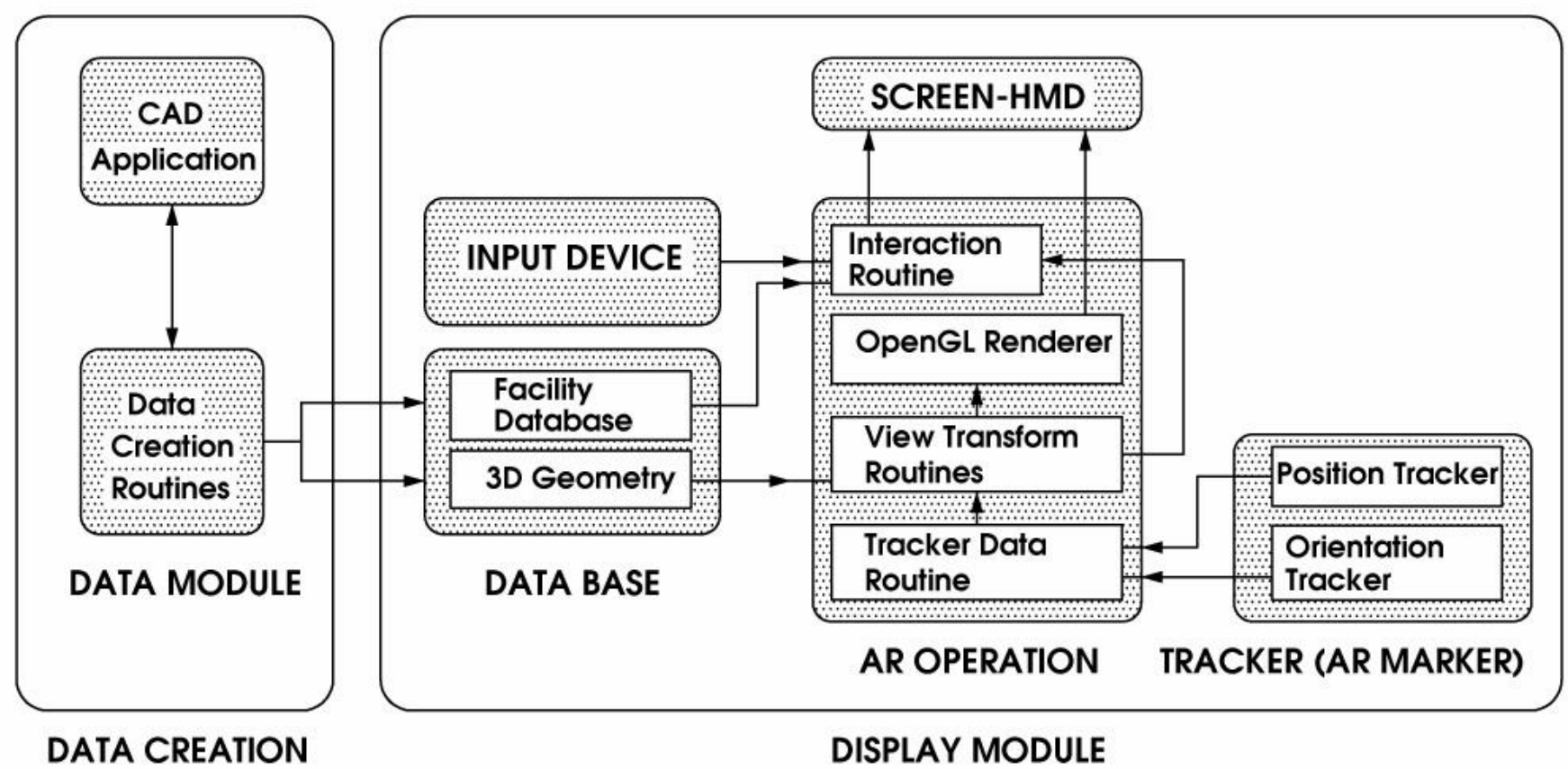

Figure 5. System diagram.

The properties of the furniture graphics are saved in a database generated by a CAD application, e.g. 3DSMax software, while OpenGL renders the final graphics. Plus, an ARToolKit software library is used to calculate the $3 \mathrm{D}$ positions and orientations of the virtual furniture.

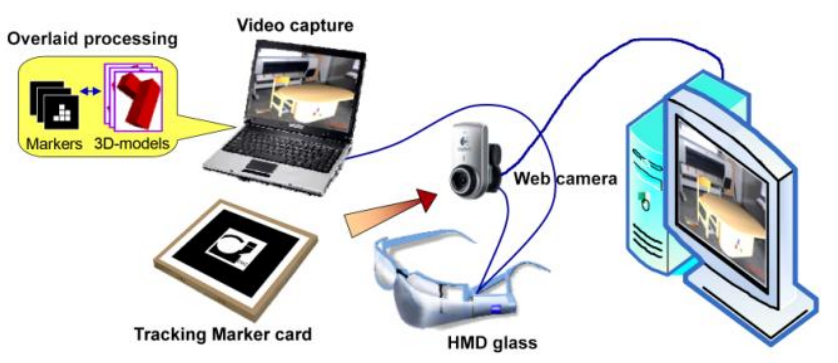

Figure 6. AR tracking\& display process: the computergenerated graphics are integrated into user's view.

\subsection{Software}

CAD applications handle the management of the building geometry data and link it to a database. Next, the AR software retrieves and displays the position and orientation data in the defined environment.

The 3DSMax or other Building Information Modelling (BIM) applications (e.g. ArchiCAD, Revit etc.) are used as the basic software for the CAD applications and also provides customized support for ARToolKit- 2.72.1. The 3DSMax (and others) produces a VRML file of a model which has a type *.wrl extension. An ARToolKit library then assumes the role of building the AR application. One of the key difficulties involved in developing an AR application is tracking the user's viewpoint. In order to determine which viewpoint to use to align the virtual imagery with real-world objects, the AR application first needs to determine the viewpoint of the user in the real world.
ARToolKit software uses computer vision algorithms to solve this problem. An ARToolKit video tracking library defines the virtual camera position and orientation relative to physical markers in real time. The ARToolKit library- the product of HIT Lab NZ- is then used to display the virtual objects.

\subsection{Hardware}

In the present study, the AR system is based on a regular PC with a Windows XP operating system running on an Intel (R) Core(TM) Quad CPU Q6600 with 2GB of RAM. Plus, a webcam, Logitech Quickcam Vision Pro, is used to capture the sense images. The user's camera is capable of detecting known patterns from a single image and calculating the $3 \mathrm{D}$ position and orientation for world-space. The virtual objects (furniture, partitions, walls, doors, etc.) are then superimposed based on marker tracking.

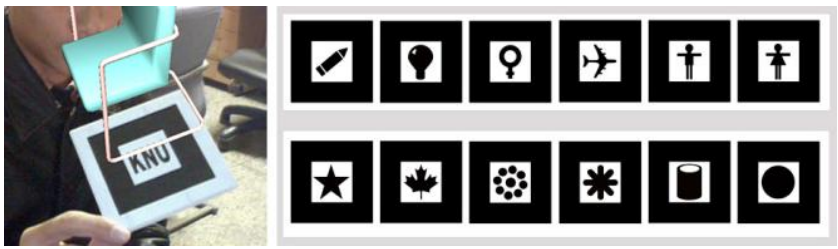

Figure 7. Left: Fiducial marker patterns. Right: Sub-marker card for Tangible AR control.

Some of the tracking markers used by an ARToolKit library are very precise and robust. In this study, $m k \_$patt.exe files were used to generate various image markers from a blankPatt.gif pattern directory. For implementation, several marker patterns and submarker templates for Tangible AR were made beforehand.

The Head-Mounted Display (HMD) also is equipped for user in the practical implementation. By using HMD for AR display, the user can move freely around virtual furniture when they are viewing it. The figure 8 shows an example of how the components 
of AR system working together to produce the final results on video eye-monitors.

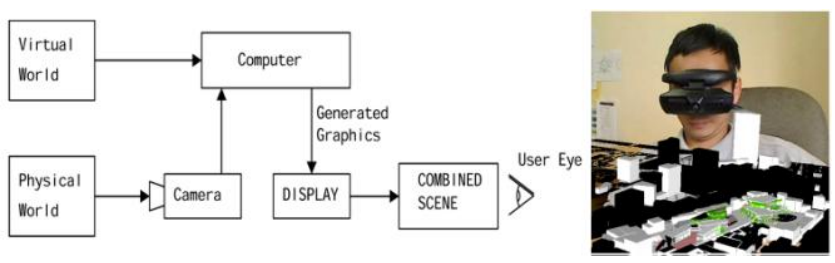

Figure 8. Schematic illustration of video overlaid- based Augmented Reality\& VR Pro AR 800x600 HMD.

\subsection{Interaction method on occlusion markers for Tangible AR}

This paper applies an interaction object- centered view to 2D interactions, which is easy to apply to Tangible AR environments where natural interaction methods are vital. In the real world, humans are able to use a variety of objects or bare fingers as a pointer. In addition, for some situations with multiple participants or bi-manual interactions, the interaction can even involve multiple pointers.

Detecting pointers over an interaction object can be achieved in numerous ways, where detecting the occlusions of the tracked object is a passive way to detect pointing actions. The occlusions of an interaction object can be easily utilized as an interaction method in Tangible AR environments in which a camera is already available for providing real-world views to the user and tracking the objects of interest with passive formal markers.

For occlusion detection, predefined formal markers are widely used for tracking real objects in Tangible AR environments. Vision-based tracking systems usually require multiple markers for tracking one object. A number of markers are attached to a single object in a pre-configured spatial relationship. In this way, the object can be tracked successfully even if some of markers in the marker set are not visible. In addition, because the spatial relationships of all the markers are known, the poses of markers that are not visible can be estimated using the markers that are recognized.

A simple way to guarantee that a marker is within the view volume is to check the visibility of its neighboring markers, referred to as boundary markers, while a marker being checked for occlusion is referred to an interaction marker.

To guarantee that an interaction marker is within the view volume, the boundary markers must be carefully placed. The convex hull of the boundary markers must include the interaction marker. For instance, for a single interaction marker, at least 2 boundary markers are needed surrounding the interaction marker (Figure 9). By checking whether these boundary markers are visible, the interaction marker can be guaranteed to be within the view volume, making it occluded if it is not detected.
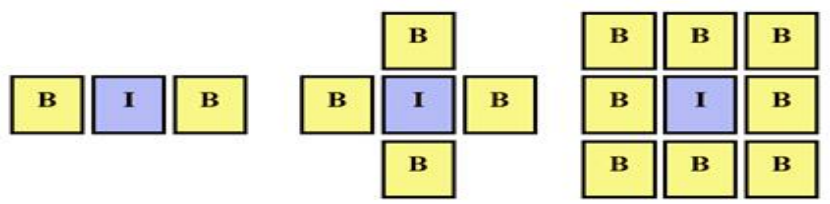

Figure 9. Boundary markers around interaction markers.
When multiple interaction markers are placed in a line, the neighbors of the interaction marker being tested can also be treated as boundary markers. These markers are referred to as hybrid markers (Figure 10). The tested marker within the view volume whenever there is at least one visible boundary (or hybrid) marker on each side. Thus, hybrid markers act as both boundaries and an interaction point. In this way, the occlusion of multiple consecutive markers can also be detected, as well as allowing the boundary markers to be out of view.

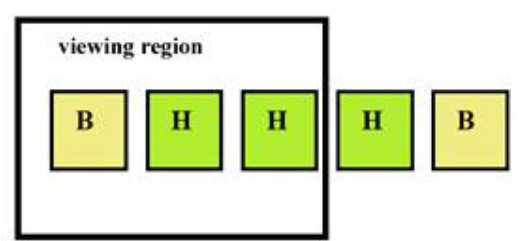

Figure 10. Hybrid markers: center hybrid marker plays role of boundary marker for left hybrid marker.

Although the boundary marker method is simple to implement and works reliably, marker wastage is unavoidable, since additional non- interactable boundary markers are required. Plus, interaction is little difficult, as the user has to make sure that enough boundary markers are within the current view.

\subsection{Interaction virtual furniture using}

\section{Tangible AR}

Tangible Augmented Reality interfaces combine a tangible user interface and augmented reality technology. In the present study, virtual furniture is modified using an occlusion- based interface for Tangible AR effects. Tangible AR interfaces are where each virtual object is registered to a physical object, and a user interacts with the virtual objects by manipulating the corresponding physical objects. In this case, occlusion is a simple way of completing interactions based on hiding the formal markers from being tracked. In this study, two sub-marker band cards are made, where one controls the color and the other controls the material of the virtual furniture. In particular, these marker templates are combined from several unit markers (Figure 7-Right).

Each unit marker corresponds to one option. In the implemented AR system, the user takes first sub-marker band card to create a virtual chair. The user can hide one unit marker using one finger. A new corresponding color is then assigned to the virtual chair. Next, the user moves the second sub-marker band card to connect with the first one in order to adjust the color volume. The corresponding virtual color slide is then added as an overlay for this band card. Changing the effect of the virtual color slide can be redefined based on the position of the hidden unit marker with the user's finger. Thus, the effect of changing the color of the virtual chair corresponds with the hidden point situation of the virtual color slide being shown.

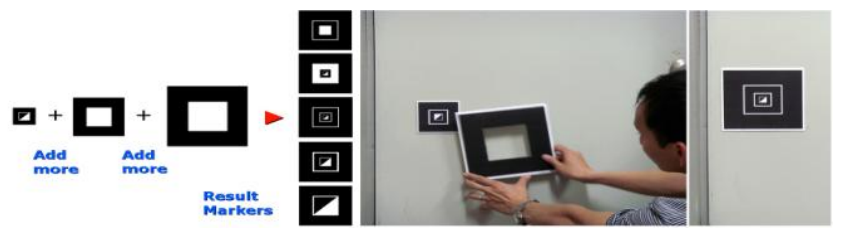

Figure 11. Left: Multi-class marker prototype; Right: User assembling separate parts of Multi-Class marker. 
This study also uses a control method based on Multi-Class marker (Figure 11- Left), which is explored based on an identifiable ability image fiducial marker in the computer-vision process. In fact, a Multi-Class marker combines the functions of several unit marker values. The operating control of a Multi-Class marker is through user assembling unit markers. Theoretically, several unit marker values can be created within one Multi-Class marker. Yet, in this study, one Multi-Class marker consists of six unit marker values. The cause of this limit is the graphic identifiable ability of a web camera. In the present study, the Multi-Class marker covers the entire virtual graphics of the AR operation, and the user can also order more furniture when more unit marker classes are fixed.

The next section shows the interaction with virtual furniture to produce a Tangible AR effect with an AR interface.

\section{IMPLEMENTATION}

The operation of the system is described in the following paragraphs. First, the user prints out the markers that will be used, where the style and size of the markers can be defined from the user interface in order to adapt it to the environment (i.e. viewing distance and size of the room). As the user walks around the room, they take a series of capture marker images with a digital camera. These marker images are then upload to the AR software as the marker tracking stage. Thereafter, the furniture augmenting system is started.

The system includes functions for handling images, moving wrlmodels and re-sizing them, and defining marker properties and threshold values for manipulating objects. The user selects different pieces of (virtual) furniture from the object list on the left, then adds, deletes, or modifies the properties, and hides them as required. Each object first appears on the marker card, however,
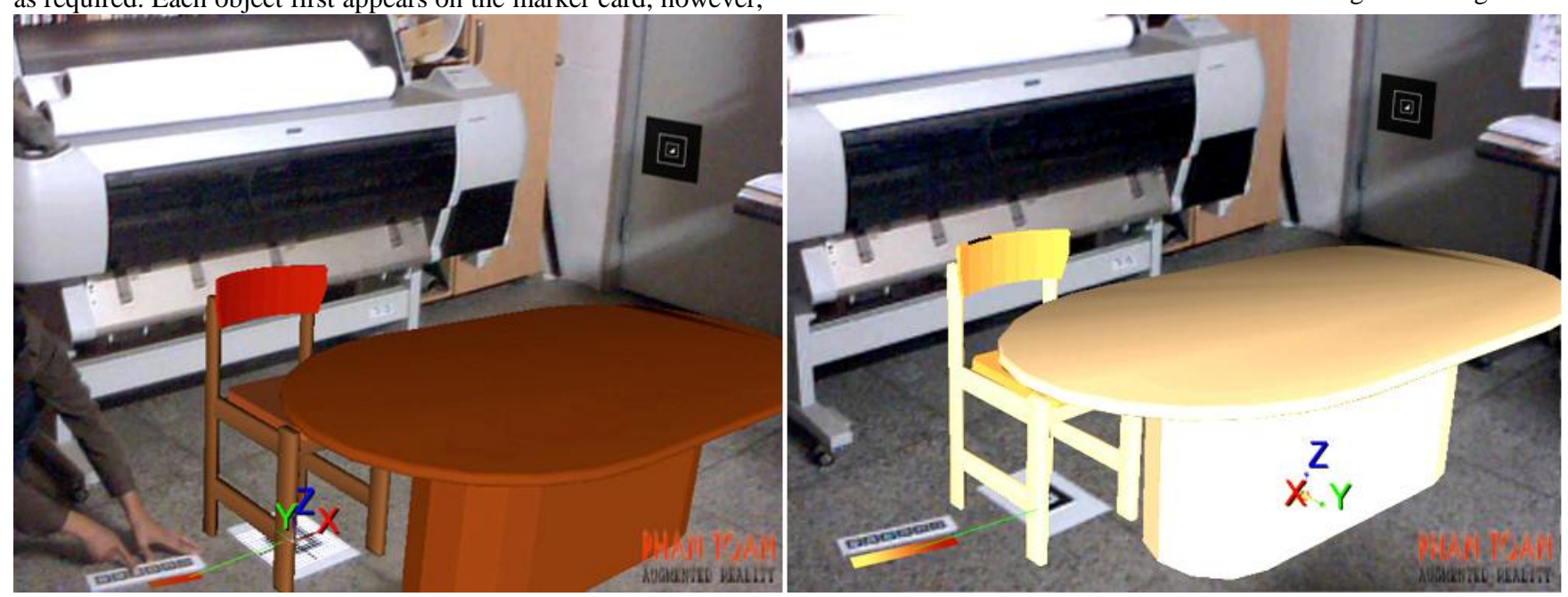

the user also can move an object to the desired position by dragging it with a mouse, or modify the threshold values of the coordinates. Many AR applications use fixed directions in the marker coordinates. As a result, when looking from an opposite direction, the object is moved to an unnatural direction. In contrast, the proposed approach is more natural for user manipulation, as no knowledge is required of the marker coordinates. First, a virtual chair and meeting table are assigned as the main samples in the present AR experiment. Once the virtual furniture has been arranged, the user can adjust the scale using digital images on the screen or a control marker band through a Tangible AR effect. In the AR photos, the user keeps the control marker template in their hand as they approach the virtual furniture. The virtual adjusted slide appears on the control marker template, allowing the user to interact with the virtual furniture through manipulating the control marker band (unit markers must be hidden using the fingers).

In another phase, if the user wants to place a sample partition in the room, the user can order a virtual partition to appear in the appropriate position. Yet, difficulties occur when the camera has difficulty viewing the tracking markers in the case of virtual and real furniture being added in the same space. With the proposed system, the implementation allows the user to change the three dimensional relationship between the virtual furniture and the marker images. Thus, the user can place a marker in any free position so that the camera can view it clearly. As with all virtual furniture, the properties of a partition or any virtual furniture can be modified in real time using the Tangible AR effect.

All the images and virtual models are loaded onto the system dynamically. Furthermore, the state of the virtual furnishing design can be saved in a project file, which can then be loaded later when the user decides to continue working their design.

Figure 12. Two-phase AR scene- user adjusts color of virtual furniture 

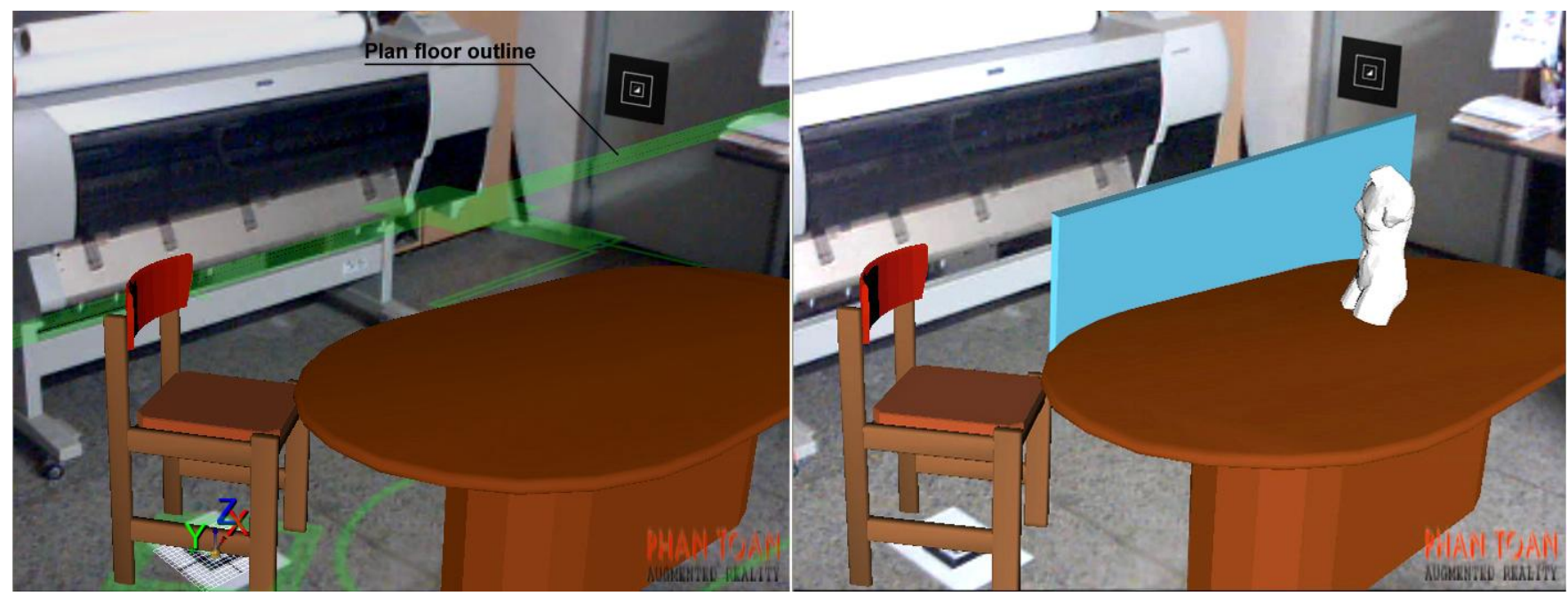

Figure 13. Chair base is moved on floor plan graphic\& the statue is added on table using AR interior design interface.

\section{CONCLUSION}

This research examined virtual furniture and adjustment work to create a new design method using Augmented Reality technology for Interior Design education.

In particular, AR technology opens up many new research fields in engineering and architecture. In an AR environment, design work can become more lively, convenient, and intelligent. Plus, design work and manufacturing can be conducted at the same time and we close relationship with each other. With AR, the virtual products of graphic technology are not only for simulation but also obtain real higher values.

Furthermore, AR technology can become a new animated simulation tool for interior design, allowing the user to see a mixed AR scene through HMD, video display, or PDA. It is also anticipated that the interactive potential can be increased according to the user's needs.

\section{ACKNOWLEDGMENTS}

This research was supported by Basic Science Research Program through the National Research Foundation of Korea (NRF) funded by the Ministry of Education, Science and Technology (2010-0015999).

\section{REFERENCES}

[1] Milgram, P., Takemura, H., Utsumi, A., and Kishimo, F. 1994. Augmented reality: A class of displays on the realityvirtual continuum. In Proceedings Telemanipulator and Telepresence Technologies: 2351-34, Retrieve 2007-03-15.

[2] Azuma, R. 1997. A survey of Augmented Reality. InPresence: Teleoperators and Virtual Environment 6, 4 (August 1997), 355-385.

[3] Woodward, C., Lahti, J., Rökkö, J., Honkamaa, P., Jäppinen, J., Rainio, K., and et al. 2007. Virtual and augmented reality in the digital building project. International Journal of Design Science and Technology, Vol.14, No.1, 23-40.
[4] Billinghurst, M., Kate, H., and Proupyrev, I. 2001. The MagicBook-Moving Seamlessly between Reality and Virtual IEEE Computer Graphics and Applications, Vol.21, No.3, 24.

[5] Siltanen, S., and Woodward, C. 2006. Augmented interiors with digital camera images. In Proceedings of Seventh Australian User Interface Conference, Australia CRPIT, 3336.

[6] Pasman, W., Woodward, C. 2003. Implementation of an Augmented Reality System on a PDA. Symposium of Mixed and Augmented Reality, ISMAR 2003, Tokyo, Japan.

[7] Phan, V. T., Choo, S. Y. 2010. A Combination of Augmented Reality and Google Earth's facilities for urban planning in idea stage. International Journal of Computer Applications, Published by Foundation of Computer Science, USA, vol. 4, No. 3, 26- 34.

[8] Sherman, W. and Craig, A. 2003. Understanding Virtual Reality: Interface, Application and Design. Morgan Kaufman Publisher.

[9] Kensek, K., Noble, D., Schiler, M. and Triparthi, A. 2000. Augmented Reality: An application for architecture. In Proceedings $8^{\text {th }}$ International Conference on Computing in Civil and Building Engineering, ASCE, Stanford, CA, 294301.

[10] Dias, J. M. S., Santos, P., Nande, P. 2003. In Your Hand Computing: Tangible Interfaces for Mixed Reality. In Proceedings of $2^{\text {nd }}$ IEEE International Augmented Reality ToolKit Workshop, Waseda University, Tokyo, Japan.

[11] Vallino, J. R. 1998. Interactive Augmented Reality. Doctor of Philosophy Thesis, University of Rochester, New York. 